\title{
Toward Climate-Resilient Development \\ First Decade with the Global Framework for Climate Services
}

\author{
Adapted from "Making Society \\ Climate Resistant: International \\ Progress under the Global \\ Framework for Climate Sciences," \\ by Chris Hewitt (Met Office), Erica \\ Allis, Simon Mason, Meredith \\ Muth, Roger Pulwarty, Joy \\ Shumake-Guillemot, Ana E. Bucher, \\ Manola Brunet, Andreas M. Fischer, \\ Angela M. Hama, Rupa Kumar Kolli, \\ Filipe Lucio, Ousmane Ndiaye, and \\ Barbara Tapia. Published online in \\ $B A M S$, February 2020. For the full, \\ citable article, see: DOI:10.1175 \\ /BAMS-D-18-0211.1.
}

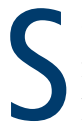
ociety has yet to optimally benefit from available climate information. While weather services routinely help weather-sensitive decision-making, similar services for decisions on longer climate time scales are less well established. Many organizations are now actively developing climate services, and a growing number of decision-makers are keen to benefit from such services.

The third World Climate Conference (WCC-3) in 2009 established the Global Framework for Climate Services (GFCS) to help society better manage the risks of climate variability and change, and adapt to and mitigate climate change, by utilizing science-based information and prediction in planning, policy, and practice. Climate services-the provision and use of climate informationhave made significant progress since WCC-3. We describe successes, challenges, and potential solutions in this worldwide effort with a focus on the role of the GFCS.

\section{Ten year's progress}

Following a review of the progress in implementing the GFCS over the past 10 years, the World Meteorological Congress established a Climate Coordination Panel in 2019 to oversee and coordinate further implementation of the GFCS under the Framework's guiding principles, including prioritizing capacity development in developing countries vulnerable to climate impacts, promoting the 
free and open exchange of climate-relevant data where possible, providing climate services primarily for the public good, and facilitating and strengthening existing activities. A cornerstone of these principles has been to build partnerships involving stakeholders. Such partnerships are essential for developing, delivering, and using climate services, and for creating a thriving market.

Challenges to the delivery and uptake of climate services include (1) inadequate availability and quality of climate data in many countries; (2) a need to create or improve access to climate services in almost all countries, and many actors lack financial resources or incentives to participate; (3) limited climate literacy and capacity to deal with climate-related risks in many countries and sectors; (4) limited experience and motivation of users and service providers to collaborate with each other; and (5) ensuring active engagement from the broad range of key organizations.

The climate information that underpins the services includes observations and monitoring of the climate, seasonal-to-annual predictions, and multidecadal projections, essential components of the landmark Paris Accord signed in 2016. Since its inception, the GFCS has focused on agriculture and food security, disaster risk reduction, health, water resources, and more recently, energy as key sectors. More than 40 developing countries have identified weather and climate services as a key to their resilient development and their ability to commit to the Paris Accord and make climate issues central to their development plans, especially in the GFCS's key sectors.

The growth in demand for climate services requires an increase in sector-tailoring and end-user-focus, as well as coordination to avoid fragmentary, contradictory implementation. The GFCS is a much-needed mechanism to provide a credible, accessible, and integrative platform for enhancing coordination and for guiding and supporting climate services worldwide. It has been an effective catalyst for establishing and strengthening regional and national capacities and structures.

\section{GFCS successes}

While the GFCS is global, societal benefits are realized typically at regional, national, and local scales. As an example, to bring together national, regional, and international climate experts, the WMO, National Meteorological and Hydrological Services (NMHSs), regional institutions, and other international organizations initiated Regional Climate Outlook Forums (RCOFs) in the late 1990s, focusing on seasonal time scales.

The primary outputs of the RCOFs were regional outlooks, which initially were consensus products for the coming season, typically in a probabilistic form based on a range of credible climate predictions. The seasonal forecasters interacted with users to identify impacts and implications and to formulate response strategies. A typical RCOF involves a training workshop on seasonal climate prediction to assist the national and regional operational climate experts, as well as special outreach sessions, involving media experts, to develop communications strategies.

A review of RCOFs conducted in 2017 included a number of recommendations to enhance their effectiveness, and the GFCS is developing the RCOF approach accordingly, supporting regional cooperation and partnership to enable useful and actionable climate information to support decisions. Recommendations included a transition to objective forecasts, standardizing operational practices, follow-up integration of seasonal outlooks in decision-making processes, "centralized" training to better target capacity, and stronger links with research.

At the national scale, National Frameworks for Climate Services (NFCS), an important concept under the GFCS, helps coordinate, facilitate, and strengthen collaboration among national institutions and other key stakeholders. This process enables NMHSs to evaluate their capacity to support decision-making and map the activities and resources needed to improve that capacity. In 2012, the GFCS piloted NFCSs in several African countries. As these NFCSs have developed, interest has grown in other countries. For example, in 2018 the Economic Community of West African States partnered with the GFCS to support its members in establishing NFCSs. As a result, Senegal has implemented its NFCS, and Benin, the Gambia, Guinea-Bissau, Guinea, Togo, Cabo Verde, and Ghana are at differing stages of formulating their own national plans.

The World Bank has used the NFCS to inform its hydrometeorological and climate change investments in several countries, as well as some of the strategic dialogues under the Pilot Programme for Climate Resilience. Similarly, the 
French Development Agency used the NFCS to inform overseas development investment. The Climate Investment Fund's Climate Risks and Early Warning Systems program has also used the NFCS approach. In Burkina Faso alone, the NFCS process has guided more than $\$ 25$ million of investment. For groups of countries, regional collaboration to enhance capacities and capabilities in service delivery are undertaken under Regional Frameworks for Climate Services, analogous and at times linked to NFCSs. The sidebar "Regional collaboration: Caribbean platform for exchange" outlines a good practice example of such regional collaboration in the Caribbean.

A different example of success at the national scale is the joint GFCS and Copernicus Climate Change Data climate data rescue through the International DAta REscue (I-DARE) portal (http://wwww.idare-portal.org/) to provide guid ance material and support data rescue in countries where the data are stored on highly perishable media.

\section{Next steps}

The GFCS is now looking to ensure sustainability with necessary financial investment in its main components and further support to climate-service delivery and uptake. A focus going forward is to improve the less mature aspects of the GFCS, in particular provideruser engagement and the Climate Services Information System. There are, however, significant remaining challenges. For example, some NMHSs are still weather-focused and have not embraced climate services, either because they do not have dedicated resources to sustain user engagement or do not have a national mandate for climate services. National service providers with insufficient capabilities undermine confidence in climate services. Other challenges include the time-consuming realities of user engagement; research capabilities that often have not been tailored for operations; the difficulties of localizing information credibly; insufficient engagement with the private sector to add value to climate service products; the partial implementation of GFCS's monitoring and evaluation framework; and the inherent limits of science in the face of climate-related shocks that cannot be fully mitigated.

Given such challenges, key strategies for climate services include:
Develop (applied) science to better match scientific capability to societal needs. The decision context and user needs lie at the heart of effective climate service uptake. The information needs to reach the right person or institution in the right form at the right time. Actionable climate services are underpinned by multidisciplinary science that considers

\section{Regional collaboration: - Caribbean platform for exchange}

In 2010, the Caribbean Institute for Meteorology and Hydrology (CIMH) and Caribbean Community Climate Change Centre (5Cs), in partnership with NOAA and the WMO, relaunched the Caribbean Regional Climate Outlook Forum (CariCOF). The CariCOF brings together meteorologists and decision-makers from various climate-sensitive sectors in the region to improve forecasts and tailoring for those sectors. Since 2012, there has been at least one forum a year, typically ahead of the wet and the dry seasons.

The Caribbean has a regional strategy for coping with climate change, approved by heads of government. In 2013, Trinidad and Tobago launched the Caribbean GFCS. Regional workshops and consultations on climate services were convened in Belize, Trinidad and Tobago, Dominica, Suriname, and Guyana. The Caribbean Council for Trade and Economic Development endorsed the GFCS in 2015.

The result is new partnerships. For example, the Early Warning Information Systems across Climate Timescales (EWISACTs) brings together the regional agencies in climate-sensitive sectors such as agriculture, public health, renewable energy, and tourism. EWISACTs helps integrate climate information and sectoral climate risk management, in part by assessing climate-related vulnerabilities and codeveloping services addressing them, and also through codelivery of user-defined climate-impact prediction. The $5 \mathrm{Cs}$ is central to developing linkages between operations to long-term adaptation and sustainability. Among other things, the $5 \mathrm{Cs}$ facilitate application of global climate model outputs to small-scale islands, provision of analytical tools, and preparation of a regional strategy for resilient development. 


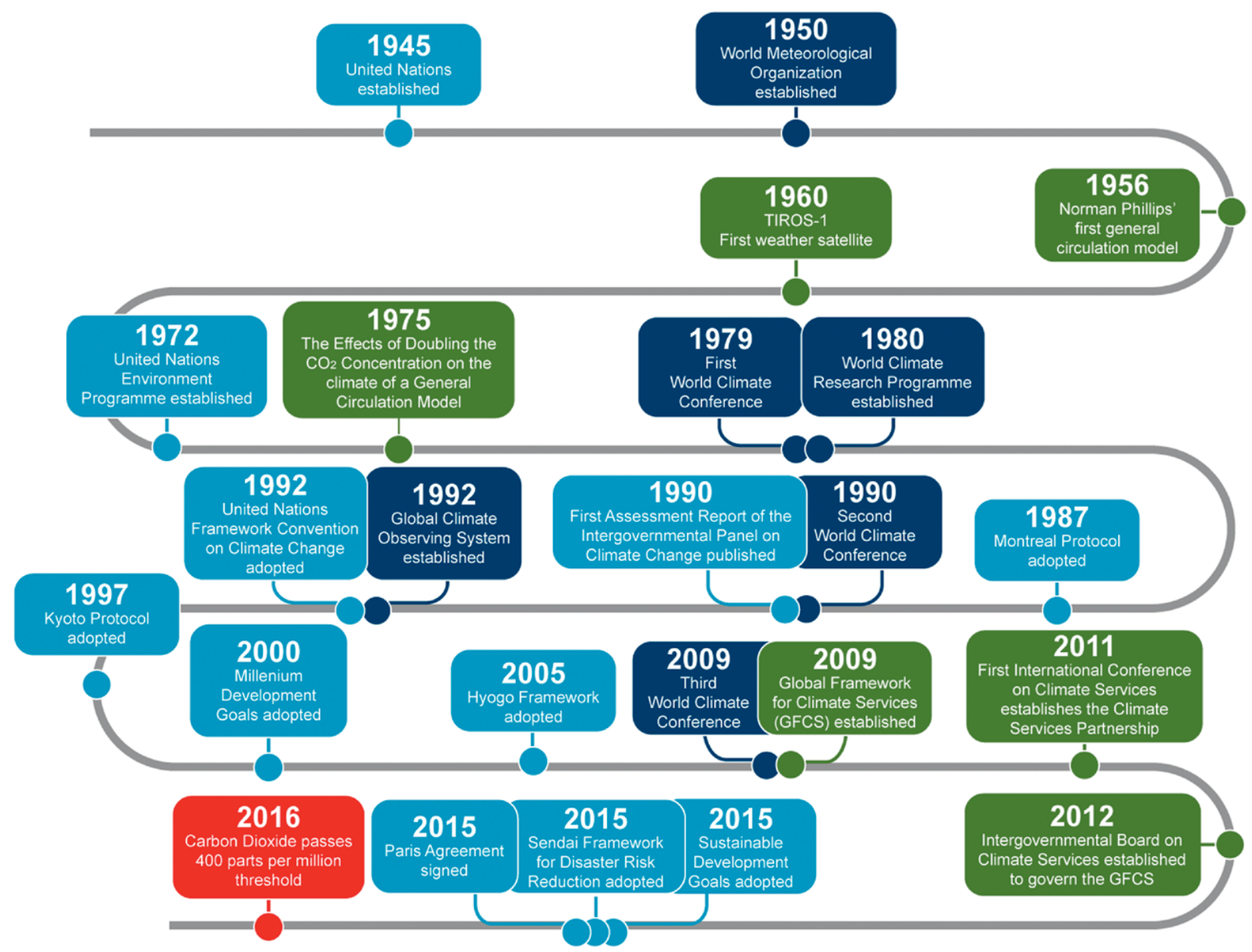

the complexity of the systems within which climate information is produced and delivered. Inclusion of social science expertise to improve understanding and translation of climate service needs, effectively engage stakeholders, and broker knowledge and understanding is essential.

\section{Enhance information management at the} global, regional, and national scales. One of the pillars of the GFCS is the Climate Services Information System (CSIS) which is the principal mechanism through which information about past, present, and future climate is archived, analyzed, modeled, exchanged and processed for use. The CSIS builds on the operational observation, modeling, and dissemination infrastructure of WMO to enable decision-relevant information through partnership.

Include climate services more widely in planning and policy. Integrating climate variability and change into development $\triangle$ Key events in the development of climate information for decision-making. planning requires systematic accounting for climate risks and opportunities at every level of dialogue, policy planning, governance, investment design, implementation, and evaluation of actions to demonstrate effectiveness. Making climate services more accessible will increase the likelihood of their uptake and use by decision-makers.

Enhance capacity and governance to enable climate services. A recent survey identified a serious capacity gap in many NMHSs. Updating regional and national training strategies in coordination with development partners and recognized centers of excellence (such as WMO Regional Training Centers) may make inroads into this gap.

Enhance monitoring, evaluation, and knowledge management and communication. Common understanding of climate services is not yet widely established. Many practitioners have little idea what climate 
services are, or that they are more than simply data and are beyond weather services. Therefore, communication strategies are needed. In addition, many people ask, "What value do climate services provide for my decision?" Responding to this question requires monitoring and evaluating the costs and benefits of climate services-a complex practice not widely undertaken.

Foster strategic partnerships and catalyze innovation. The international development community is committed to partnering with the climate-service community. This commitment increasingly facilitates climate-resilient and low-carbon development. Numerous projects funded by development agencies (such as the World Bank, the U.S. Agency for International Development, and the European Commission) actively support climate resilience, climate-risk management, and climate change adaptation. To succeed, these climate-smart initiatives require timely and accessible climate services. The role of the private sector also needs to be considered, for example to innovate and add value to products.

\section{Conclusion}

The GFCS has helped build and evolve the global development, coordination, and delivery of climate service activities, and has created a national service-oriented culture within the context of NFCSs, allowing a range of sectors to engage in climate services within many countries. Regional Frameworks for Climate Services are being developed to support regional collaboration and partnership to enhance capacity in service delivery, with links to the NFCSs at the national scale.

Climate services are successfully being used by a growing number of decision-makers in both the public and private sectors. Ensuring that the beneficiaries of the services are better involved will drive user-focused development of scientific and technical capability. Such developments will ultimately enable better risk management and climate-smart decisions. Those engaging in climate services are ideally placed to serve as a focus for climate-change adaptation and mitigation, and the GFCS is the international coordination and facilitation needed to help society better manage risks and opportunities arising from climate variability and climate change.
BAMS: What would you like readers to learn from this article?

Rupa Kumar Kolli (WMO): Sustained efforts to build and mainstream climate services are necessary for a resilient society.

Barbara Tapia (Chilean Meteorological Service): It is important to be informed with clear and robust climate information to make better decisions.

\section{Manola Brunet (University Rovira i Virgili/University of East Anglia): There's a need for progress in the production and delivery of robust and timely climate services.}

Chris Hewitt: However, there are major challenges developing, providing, and using climate services. Coordinated international efforts are helping address the challenges.

BAMS: Essentially, the story of the GFCS seems to be that story of international coordination.

\section{Simon Mason (International Research Institute for Climate and Society): Without global coordination and coopera- tion, climate services devel- opment would be a chaotic mess.}

BAMS: Coordination and cooperation on that scale is a major undertaking. What have been disappointments, so far, in the development of climate services?

Erica Allis (WMO): Lack of standards, lack of strategic 
leadership, lack of transparency in skill assessments to enable users to make decisions based on the information.

SM: Continued focus on tercile-based probabilistic forecasts of seasonal rainfall totals.

\section{Ousmane Ndiaye (National} Civil Aviation and Meteorology Agency, Senegal): The lack of work on demand-driven products and lack of sustainable funding for and ignorance (decaying) of the observing network.

BT: That almost two decades have been needed to establish the GFCS in the Americas.

\section{Andreas Fischer and Michi- ko Hama (MeteoSwiss):}

Despite all the progress in developing and tailoring user-centric climate services and the urgency of taking on the challenges, global greenhouse gas emissions are still on the rise without any sign of slowdown.

BAMS: Why has progress been slow?

$\boldsymbol{A F}$ and $\boldsymbol{M H}$ : The concept of climate services has not yet become a household name even though they are THE prerequisite for successful and low-regret adaptation and mitigation.

CH: Engaging with beneficiaries, and potential beneficiaries, of the services is an ongoing challenge. I don't feel that the whole community (developers, providers, users of the services) has properly exploited the available climate information and knowledge yet.

\section{Ana Bucher (World Bank):}

There's a lack of understanding of the value climate services can have in supporting decision-making. We need to ensure we can demonstrate and monetize the value of such services in our daily lives.

RKK: There's also slow progress in the development of the user interface platform, and inadequate two-way partnerships between users and providers at all levels.

BAMS: What have been the most satisfying successes of the GFCS?

$\mathbf{C H}$ : Over the past few years, seeing just how many countries now adopt the GFCS and its concepts.

BT: To see the launch of the two Regional Climate Centers in South America.

RKK: These RCCs and RCOFs spearhead regional collaboration in a sustainable manner.

$\boldsymbol{A B}$ : Integration of science and societal stakeholders, including development partners.

$\boldsymbol{A F}$ and $\mathbf{M H}$ : It was and is satisfying to see the increase of commitment and collaboration across sectors-more and more sectoral partners have jumped or are about to jump on the "climate services" bandwagon.

SM: Compare the state of climate services generally as they are now to how they were 30 years ago. With patience, old practices and ways of thinking can be modified.

BAMS: What are some of the challenges for climate services and the GFCS?

ON: The availability of data, computational power, sharing platform, and open-source software.

$\boldsymbol{A F}$ and $\mathbf{M H}$ : Scientists and users have joined forces considerably. By now most parties accept that sustainable solutions for practitioners need to be coproduced to be successful. However, full-fledged participatory approaches from start to end of the cycle are still rare.

$\boldsymbol{A B}$ : Internalization of the value of predicting weather and understanding potential changes in the future. The weather and climate community was successful in demonstrating how this information can affect decision-making for our daily lives.

BAMS: What expectations did you have for the future impact of GFCS?

CH: Now that the GFCS is being adopted as a useful framework in so many regions and by so many countries around the world, I hope that this momentum will be maintained and we will see many of the challenges addressed and climate information used more and more to better inform decisions to ensure society benefits. 